JOURNAL OF THE

AMERICAN MATHEMATICAL SOCIETY

Volume 16, Number 3, Pages 681-704

S 0894-0347(03)00419-3

Article electronically published on January 3, 2003

\title{
MULTIVARIABLE COCHAIN OPERATIONS AND LITTLE $n$-CUBES
}

\author{
JAMES E. MCCLURE AND JEFFREY H. SMITH
}

\section{INTRODUCTION}

This paper has two goals. The first (see Theorem 2.15 and Remark 2.16(a)) is to construct a small $E_{\infty}$ chain operad $\mathcal{S}$ which acts naturally on the normalized cochains $S^{*} X$ of a topological space $X$. This is of interest in view of a theorem of Mandell [15, page 44] which states that if $\mathcal{O}$ is any $E_{\infty}$ chain operad over $\overline{\mathbb{F}}_{p}$ (the algebraic closure of the field with $p$ elements) which acts naturally on $S^{*} X \otimes \overline{\mathbb{F}}_{p}$, then the homotopy category of connected $p$-complete nilpotent spaces of finite type imbeds in the homotopy category of $\mathcal{O}$ algebras; tensoring our operad with $\overline{\mathbf{F}}_{p}$ gives an operad to which Mandell's theorem applies. Operads which act naturally on the normalized cochains of spaces were already known to exist [6], 9], but the known examples are uncountably generated and the structure maps are hard to describe explicitly, whereas our operad is of finite type and has a perspicuous description (see Definition 2.14 and Propositions 2.18, 2.19] and 2.26).

The second goal (see Theorem 3.5) is to give, for each $n$, a suboperad $\mathcal{S}_{n}$ of $\mathcal{S}$ which is quasi-isomorphic, in the category of chain operads over the integers, to the normalized singular chain operad of the little $n$-cubes operad (this is the smallest and simplest known chain model for the little $n$-cubes). One reason this is interesting is that the operad $\mathcal{S}_{2}$ acts naturally on the normalized Hochschild cohomology complex of an associative ring (see Theorem 4.1), so we obtain a solution of Deligne's Hochschild cohomology conjecture [5]. Other solutions of Deligne's Hochschild cohomology conjecture are known (12, 13, 17, 21, 22, 23]) but the one we give here is significantly shorter than the previously known solutions. Also, our solution (like those in [17, 13]) is valid over the integers and not only in characteristic 0 . We expect that Theorem 3.5 will lead to one way of proving the generalized version of Deligne's conjecture proposed by Kontsevich in [12].

The operad $\mathcal{S}_{2}$ is isomorphic to the operad $\mathcal{C}$ considered in our previous paper [17], but the description we give here is more explicit and the proof that $\mathcal{S}_{2}$ is quasiisomorphic to the normalized singular chains of the little 2-cubes is much simpler than the corresponding proof in 17 .

The organization is as follows: in Section 2 we define the chain operad $\mathcal{S}$ and state Theorem 2.15 and Propositions 2.18, 2.19 and 2.26. In Section 3 we define

Received by the editors June 25, 2001 and, in revised form, June 28, 2002.

2000 Mathematics Subject Classification. Primary 18D50; Secondary 55P48, 16E40.

The first author was partially supported by NSF grant DMS-9971953. He thanks the Lord for making his work possible.

The second author was partially supported by NSF grant DMS-9971953. 
the chain operads $S_{n}$ and state Theorem [3.5. In Section 4 we describe the action of $\mathcal{S}_{2}$ on the Hochschild complex. Section 5 gives the proof of Theorem 3.5, using a method due to Berger [2], and Sections 6 7 and 8 give the proofs of Propositions 2.18 , 2.19 and 2.26

The results of Section 2 have been discovered independently (but with a different choice of signs) in the joint work of Berger and Fresse [3]. After learning Theorem 3.5 from us, they have found a different proof of it using their methods.

\section{THE CHAIN OPERAD $\mathcal{S}$}

Our goal in this section is to define a family of cochain operations which generate a small $E_{\infty}$ chain operad $\mathcal{S}$ and to give the formulas which describe the structure of $\mathcal{S}$.

First we need to specify what we mean by cochains. We write $S_{*} X$ for the normalized singular chains of a space $X$ (that is, the singular chains modulo degeneracies; the reason for using normalized chains is given in the proof of Lemma 2.12).

Definition 2.1. Let $\mathbb{Z}[0]$ denote $\mathbb{Z}$ considered as a chain complex concentrated in dimension 0 . The normalized singular cochain complex $S^{*} X$ is the cochain complex $\operatorname{Hom}\left(S_{*} X, \mathbb{Z}[0]\right)$.

Note that with this definition the coboundary $d$ satisfies

$$
d(x)=-(-1)^{|x|} x \circ \partial
$$

which differs by a sign from the usual coboundary (see [7, Remark VI.10.28]).

Before giving the formal definitions of the cochain operations we are interested in, we begin with an informal discussion; in particular we denote all signs by \pm for the time being.

Definition 2.2. If $\sigma: \Delta^{r} \rightarrow X$, and if $a_{0}, \ldots, a_{p} \in\{0, \ldots, r\}$, we write

$$
\sigma\left(a_{0}, \ldots, a_{p}\right): \Delta^{p} \rightarrow \Delta^{r}
$$

for the affine map which takes vertex $i$ of $\Delta^{p}$ to vertex $a_{i}$ of $\Delta^{r}$.

Our first example of a cochain operation is the chain-level cup product. If $x \in$ $S^{p} X, y \in S^{q} X$, then $x \smile y \in S^{p+q} X$ is defined by

$$
(x \smile y)(\sigma)=x(\sigma(0, \ldots, p)) \cdot y(\sigma(p, \ldots, p+q))
$$

where $\sigma: \Delta^{p+q} \rightarrow X$.

Next we recall Steenrod's original definition of the cup-1 product [20, bottom of page 293]. If $x \in S^{p} X, y \in S^{q} X$, then $x \smile_{1} y \in S^{p+q-1} X$ is defined by

$$
\left(x \smile_{1} y\right)(\sigma)=\sum_{i<j} \pm x(\sigma(0, \ldots, i, j, \ldots, p+q-1)) \cdot y(\sigma(i, \ldots, j))
$$

where $\sigma: \Delta^{p+q-1} \rightarrow X$. (Some of the terms in this formula involve evaluating a cochain on a simplex whose degree does not match that of the cochain; all such terms are defined to be 0 .)

To describe the higher cup- $i$ products, we need a definition.

Definition 2.3. Let $T$ be a finite totally ordered set. An overlapping partition $\mathcal{A}$ of $T$ with $m$ pieces is a collection of subsets $A_{1}, \ldots, A_{m}$ of $T$ with the following properties: 
(a) If $j<j^{\prime}$, then each element of $A_{j}$ is $\leq$ each element of $A_{j^{\prime}}$.

(b) $A_{j} \cap A_{j+1}$ has exactly one element for each $j<m$.

Thus consecutive pieces of the overlapping partition are required to have exactly one element in common. For example, the collection $\{\{0,1,2\},\{2,3\},\{3\},\{3,4,5\}\}$ is an overlapping partition of $\{0,1,2,3,4,5\}$.

Remark 2.4. For later use we record the elementary fact that an overlapping partition $A_{1}, \ldots, A_{m}$ is completely determined by the $m-1$ elements $A_{1} \cap A_{2}, \ldots, A_{m-1} \cap$ $A_{m}$, which we will refer to as the overlap points.

Now Steenrod's original definition of the cup- $i$ product [20, top of page 294] is

$$
\left(x \smile_{i} y\right)(\sigma)=\sum \pm x\left(\sigma\left(A_{1} \amalg A_{3} \amalg \cdots\right)\right) \cdot y\left(\sigma\left(A_{2} \amalg A_{4} \amalg \cdots\right)\right)
$$

where the sum is over all overlapping partitions of $\{0, \ldots, p+q-i\}$ with $i+2$ pieces. Here $A_{1} \amalg A_{3} \amalg \cdots$ and $A_{2} \amalg A_{4} \amalg \cdots$ denote the disjoint unions, that is, repetitions are not eliminated; note that all terms for which $A_{1} \amalg A_{3} \amalg \cdots$ or $A_{2} \amalg A_{4} \amalg \cdots$ have repetitions will be zero, because the corresponding simplices will be degenerate.

There is a useful piece of notation (due to Benson [1. page 147] and Milgram, and rediscovered by us) which clarifies the situation. We write $12(x, y)$ for $x \smile y$, $121(x, y)$ for $x \smile_{1} y, 1212(x, y)$ for $x \smile_{2} y$, and so on. We also write $21(x, y)$ for $y \smile x, 212(x, y)$ for $y \smile_{1} x$ and so on. The idea is that to evaluate (for example) $12121(x, y)$ on a simplex $\sigma: \Delta^{r} \rightarrow X$, we sum over all overlapping partitions of $\{0, \ldots, r\}$ with 5 pieces (because the sequence 12121 has 5 entries) and the term corresponding to a partition $A_{1}, A_{2}, A_{3}, A_{4}, A_{5}$ is

$$
x\left(\sigma\left(A_{1} \amalg A_{3} \amalg A_{5}\right)\right) \cdot y\left(\sigma\left(A_{2} \amalg A_{4}\right)\right) ;
$$

here $A_{1}, A_{3}, A_{5}$ go with $x$ because the 1 st, 3 rd and 5 th entries of the sequence 12121 are 1's and $x$ is the 1st variable in the symbol $12121(x, y)$.

It will now be obvious that one can use a similar idea to define multilinear cochain operations with more than 2 variables (this fact was first noticed by Benson [1. page 147], who used it to give a definition of the odd-primary Steenrod operations in the cohomology of groups, and by Milgram, and later independently by us). For example, it is natural to interpret the symbol $12312(x, y, z)$ by

$$
12312(x, y, z)(\sigma)=\sum \pm x\left(\sigma\left(A_{1} \amalg A_{4}\right)\right) \cdot y\left(\sigma\left(A_{2} \amalg A_{5}\right)\right) \cdot z\left(\sigma\left(A_{3}\right)\right) .
$$

Similarly one can define a multilinear cochain operation in $k$ variables for any finite sequence with entries $1, \ldots, k$ in which each of the numbers $1, \ldots, k$ is used at least once. We will call multilinear cochain operations of this type sequence operations.

Now we turn to the formal definitions.

Notation 2.5. For $k \geq 1$ let $\bar{k}$ be the set $\{1, \ldots, k\}$, and let $\overline{0}=\emptyset$.

It will be convenient to think of sequences of length $m$ with entries in the set $\bar{k}$ as functions $\bar{m} \rightarrow \bar{k}$. The sequences in which each of the numbers $1, \ldots, k$ is used at least once correspond to surjections $\bar{m} \rightarrow \bar{k}$.

Our next three definitions are needed in order to specify the signs in our formulas.

Notation 2.6. The cardinality of a set $A$ will be denoted $|A|$, and $\|A\|$ will denote $|A|-1$. 
Definition 2.7. For each surjection $f: \bar{m} \rightarrow \bar{k}$ define $\tau_{f}: \bar{m} \rightarrow \bar{m}$ by

$$
\tau_{f}(j)=\mid\left\{j^{\prime} \in \bar{m}: f\left(j^{\prime}\right)<f(j) \text { or } f\left(j^{\prime}\right)=f(j) \text { and } j^{\prime} \leq j\right\} \mid .
$$

Remark 2.8. Any surjection $f: \bar{m} \rightarrow \bar{k}$ can be factored (usually in more than one way) as a permutation of $\bar{m}$ followed by an order-preserving map $\bar{m} \rightarrow \bar{k}$. Among permutations of $\bar{m}$ that arise in this way, $\tau$ is the only one that is order preserving on each set $f^{-1}(i)$.

Definition 2.9. Let $T$ be a finite totally ordered set. For each surjection $f: \bar{m} \rightarrow \bar{k}$ and each overlapping partition $\mathcal{A}$ of $T$ with $m$ pieces, define

$$
\epsilon(f, \mathcal{A})=\sum_{\substack{j<j^{\prime} \\ f(j)>f\left(j^{\prime}\right)}}\left\|A_{j}\right\|\left\|A_{j^{\prime}}\right\|+\sum_{j=1}^{m}\left\|A_{j}\right\|\left(\tau_{f}(j)-f(j)\right) .
$$

Now we can define the sequence operation associated to a surjection $f: \bar{m} \rightarrow \bar{k}$. We write $S_{*} X$ for the normalized singular chains of $X$.

Definition 2.10. (a) Given a surjection $f: \bar{m} \rightarrow \bar{k}$ and $\sigma: \Delta^{p} \rightarrow X$, define $\sigma[f] \in\left(S_{*} X\right)^{\otimes k}$ by

$$
\sigma[f]=\sum_{\mathcal{A}}(-1)^{\epsilon(f, \mathcal{A})} \bigotimes_{i=1}^{k} \sigma\left(\coprod_{f(j)=i} A_{j}\right) .
$$

Here $\mathcal{A}$ runs through the overlapping partitions of $\{0, \ldots, p\}$ with $m$ pieces.

(b) Given a surjection $f: \bar{m} \rightarrow \bar{k}$, define a natural transformation

$$
\langle f\rangle:\left(S^{*} X\right)^{\otimes k} \rightarrow S^{*} X
$$

by

$$
\langle f\rangle\left(x_{1} \otimes \cdots \otimes x_{k}\right)(\sigma)=(-1)^{(m-k)}\left(x_{1} \otimes \cdots \otimes x_{k}\right)(\sigma[f]) .
$$

Remark 2.11. (a) When $f: \bar{m} \rightarrow \overline{2}$ is the function corresponding to the sequence $1212 \cdots$ with $m$ entries, then $\langle f\rangle$ is (up to sign) the same as Steenrod's $\smile_{m-2}$.

(b) In particular, when $f$ corresponds to 12 , the operation $\langle f\rangle\left(x_{1} \otimes x_{2}\right)$ is the same as Dold's definition [7, page 222] of the cup product $x_{1} \smile x_{2}$, which differs from the usual definition by the sign $(-1)^{\left|x_{1}\right|\left|x_{2}\right|}$.

(c) For all $f,\langle f\rangle$ lowers the total degree by $m-k$.

(d) To explain the sign in part (b) of Definition 2.10, we first note that the degree of $\sigma$ must be $\left(\left|x_{1}\right|+\cdots+\left|x_{k}\right|\right)-(m-k)$ in order for the left-hand side to be nonzero. Now $\langle f\rangle$, which has degree $m-k$, is being moved past $x_{1}, \ldots, x_{k}$ and $\sigma$, so the sign is $(-1)^{(m-k)^{2}}=(-1)^{m-k}$.

(e) There are other ways to choose the sign in part (a) of Definition 2.10 but the choice we have given seems to lead to the simplest signs in other formulas (such as those in Propositions $2.18,2.19$ and 2.26) that depend on this one. Our choice of signs is motivated by the cosimplicial point of view developed in [18.

(f) Here is a conceptual way to keep track of the signs: we associate to $f$ the product of simplices $\prod \Delta^{\left\|f^{-1}(i)\right\|}$, and we represent the $\left\|f^{-1}(i)\right\|$-dimensional simplex $\Delta^{\left\|f^{-1}(i)\right\|}$ by a sequence of $\left\|f^{-1}(i)\right\|$ one-dimensional objects (each denoted by $*$ ). The sign in part (a) of Definition 2.10 comes from permuting the simplices $\sigma\left(A_{j}\right)$ and then moving the $*$ 's corresponding to $\Delta^{\left\|f^{-1}(i)\right\|}$, in order, into the positions between the pieces of $\sigma\left(\coprod_{f(j)=i} A_{j}\right)$. 
Next we observe that $\langle f\rangle$ will be identically zero for certain $f$.

Lemma 2.12. If $f(l)=f(l+1)$ for some $l \in \bar{m}$, then $\langle f\rangle=0$.

Proof. It suffices to show that $\sigma[f]=0$ for all $\sigma: \Delta^{p} \rightarrow X$. Let $i_{0}=f(l)=f(l+1)$. If $\mathcal{A}$ is any overlapping partition of $\{0, \ldots, p\}$ with $m$ pieces, then (since $A_{l}$ and $A_{l+1}$ have an element in common) $\coprod_{f(j)=i_{0}} A_{j}$ will have a repeated entry and therefore the simplex $\sigma\left(\coprod_{f(j)=i_{0}} A_{j}\right)$ will be degenerate, which means it will represent 0 in the normalized chains $S_{*} X$.

This motivates:

Definition 2.13. A function $f: \bar{m} \rightarrow \bar{k}$ is nondegenerate if it is surjective and $f(j) \neq f(j+1)$ for $1 \leq j<m$. Otherwise $f$ is degenerate.

In particular, the identity map of $\overline{0}$ is nondegenerate. The sequence corresponding to a nondegenerate function has adjacent entries distinct.

Definition 2.14. Let $\mathcal{S}(k)$ be the graded abelian group freely generated by the maps $f: \bar{m} \rightarrow \bar{k}$ (where $f$ is assigned the degree $m-k$ ) modulo the subgroup generated by the degenerate maps.

The symbol $\mathcal{S}$ stands for "sequence operad." Note that $\mathcal{S}(k)$ is freely generated by the nondegenerate $f: \bar{m} \rightarrow \bar{k}$.

Now let $\mathcal{N}(k)$ be the graded abelian group of natural transformations

$$
\left(S^{*} X\right)^{\otimes k} \rightarrow S^{*} X
$$

where a transformation $\nu$ is assigned degree $n$ if it lowers total degree by $n$. Definition 2.10 gives a homomorphism $\mathcal{S}(k) \rightarrow \mathcal{N}(k)$ which is easily seen to be a monomorphism. Each $\mathcal{N}(k)$ is a chain complex, with differential

$$
\partial \nu=d \circ \nu-(-1)^{|\nu|} \nu \circ d
$$

where $d$ is the coboundary of $S^{*} X$ (resp. $\left.\left(S^{*} X\right)^{\otimes k}\right)$. Moreover, the collection $\mathcal{N}$ of all the $\mathcal{N}(k)$ with $k \geq 0$ is a chain operad, where the action of the symmetric group on $\mathcal{N}(k)$ and the multivariable composition operations are the obvious ones. $\left(\mathcal{N}\right.$ should be thought of as the endomorphism operad of the functor $S^{*}$.)

Let $\mathcal{S}$ be the collection of all $\mathcal{S}(k)$ with $k \geq 0$. Our main theorem in this section is:

Theorem 2.15. (a) $\mathcal{S}(k)$ is a subcomplex of $\mathcal{N}(k)$.

(b) $\mathcal{S}$ is a sub-chain-operad of $\mathcal{N}$.

(c) Each $\mathcal{S}(k)$ has the homology of a point.

Remark 2.16. (a) Part (c) of the theorem says that $\mathcal{S}$ is an $E_{\infty}$ chain operad. Thus $\mathcal{S}$ is an $E_{\infty}$ chain operad which (by definition) acts naturally on $S^{*} X$. Propositions 2.18, 2.19] and 2.26 below give explicit formulas for the structure of $\mathcal{S}$.

(b) A right coalgebra over an operad $\mathcal{O}$ is a chain complex $C$ together with maps

$$
\delta_{k}: C \otimes \mathcal{O}(k) \rightarrow C^{\otimes k}
$$

which are consistent with the symmetric group action, composition and unit of $\mathcal{O}$. The maps $S_{*} X \otimes \mathcal{S}(k) \rightarrow\left(S_{*} X\right)^{\otimes k}$ which take $\sigma \otimes f$ to $\sigma[f]$ give $S_{*} X$ a natural structure of right coalgebra over $\mathcal{S}$. 
(c) Part (a) of the theorem is stated on page 147 of [1] without a proof. The same page states incorrectly that $\mathcal{S}(k)$ is not contractible, but it gives the correct chain homotopy needed to prove (c).

(d) Ezra Getzler has informed us that during the period from 1995 to 1997, after learning of the sequence operations from Milgram, he proved part (c) of Theorem 2.15 and noticed parts (a) and (b) without proving them.

The proof of Theorem 2.15 will occupy the rest of this section, with some calculations deferred to later sections.

To prove part (a), we need to show that the differential of a sequence operation is a $\mathbb{Z}$-linear combination of sequence operations. To state the precise result, we need a definition.

Definition 2.17. Given $f: \bar{m} \rightarrow \bar{k}$ and a subset $S$ of $\bar{m}$, let $s=|S|$ and write $f_{S}$ for the composite

$$
\bar{s} \rightarrow S \hookrightarrow \bar{m} \stackrel{f}{\longrightarrow} \bar{k},
$$

where the first map is the unique order-preserving bijection.

Proposition 2.18. Let $f: \bar{m} \rightarrow \bar{k}$ be nondegenerate. Then

$$
\partial\langle f\rangle=\sum_{j=0}^{m}(-1)^{\tau_{f}(j)-f(j)}\left\langle f_{\bar{m}-\{j\}}\right\rangle .
$$

(Note that, by Lemma 2.12 , the terms for which $f_{\bar{m}-\{j\}}$ is degenerate are zero.) For example, if we write $f$ 's as sequences, we have

$$
\partial\langle 12312\rangle=\langle 2312\rangle-\langle 1232\rangle-\langle 1312\rangle+\langle 1231\rangle
$$

and

$$
\partial\langle 123121\rangle=\langle 23121\rangle-\langle 12321\rangle+\langle 12312\rangle+\langle 13121\rangle .
$$

The pattern is that we delete each 1 , then each 2 , etc., and the signs alternate, except that the last term obtained by deleting $i$ has the same sign as the first term obtained by deleting $i+1$; if a term has an adjacent pair equal or if it does not contain all the numbers from 1 to $k$, it is zero. (A conceptual way to remember the signs is that they are the same as the signs in the cellular chain complex of $\prod \Delta^{\left\|f^{-1}(i)\right\|}$; cf. Remark 2.11(f).)

Here is an outline of the proof of Proposition 2.18; the complete proof is given in Section [7. By definition $(\partial\langle f\rangle)\left(x_{1} \otimes \cdots \otimes x_{k}\right)(\sigma)$ is

$$
\pm\langle f\rangle\left(x_{1} \otimes \cdots \otimes x_{k}\right)(\partial \sigma) \pm\langle f\rangle\left(d\left(x_{1} \otimes \cdots \otimes x_{k}\right)\right)(\sigma)
$$

and according to Definition 2.10 (b) this can be rewritten

$$
\pm\left(x_{1} \otimes \cdots \otimes x_{k}\right)((\partial \sigma)[f]) \pm\left(x_{1} \otimes \cdots \otimes x_{k}\right)(\partial(\sigma[f]))
$$

so the question is what is the difference between $(\partial \sigma)[f]$ and $\partial(\sigma[f])$; the statement of Proposition 2.18 says that this difference should be a sum of terms of the form $\pm \sigma\left[f_{\bar{m}-\{j\}}\right]$. When we evaluate $\partial(\sigma[f])$ using Definition [2.10)(a), we get a sum indexed over overlapping partitions $\mathcal{A}$ and, for each such partition, a sum in which the elements of the sets $\coprod_{f(j)=i} A_{j}$ are deleted in turn. Most of the terms in this double sum also occur in $(\partial \sigma)[f]$ and so they cancel in (2.1) (assuming that the signs work correctly, which they do). If one of the sets $A_{j}$ has a single element, then the term in which it is deleted does not occur in $(\partial \sigma)[f]$; instead it is a term in $\sigma\left[f_{\bar{m}-\{j\}}\right]$. The only other possibility is that $\left|A_{j}\right| \geq 2$ and the element in $A_{j}$ which 
is deleted is at the beginning or end of $A_{j}$; terms of this form occur twice each in $\partial(\sigma[f])$, and when the signs are taken into account they cancel.

We now turn to part (b) of Theorem 2.15] We must show three things: that the identity element of $\mathcal{N}(1)$ is in $\mathcal{S}(1)$, that $\mathcal{S}(k)$ is preserved by the permutation action on $\mathcal{N}(k)$, and that $\mathcal{S}$ is closed under multivariable composition. The first of these is easy: the identity element in $\mathcal{N}(1)$ is the sequence operation induced by the identity map $\overline{1} \rightarrow \overline{1}$.

Next we must calculate the effect of permutations on sequence operations. Inspection of Definition 2.10 shows at once that the composite of a permutation and a sequence operation is (up to sign) another sequence operation. Here is the precise formula:

Proposition 2.19. Let $\rho \in \Sigma_{k}$ and let $f: \bar{m} \rightarrow \bar{k}$ be nondegenerate. Then

$$
\langle f\rangle \rho=(-1)^{\zeta(f, \rho)}\left\langle\rho^{-1} \circ f\right\rangle
$$

where the sign is given by

$$
\zeta(f, \rho)=\sum\left\|f^{-1}(i)\right\|\left\|f^{-1}\left(i^{\prime}\right)\right\|
$$

with the sum taken over all pairs for which $i<i^{\prime}$ and $\rho^{-1}(i)>\rho^{-1}\left(i^{\prime}\right)$.

A conceptual way to remember the sign is that it is the same as the sign coming from the permutation of the factors in $\prod \Delta^{\left\|f^{-1}(i)\right\|}$; cf. Remark 2.11(f).

We defer the proof of Proposition 2.19 to Section 6

To complete the proof of part (b) of Theorem 2.15, we need to show that the multivariable composite of sequence operations is a $\mathbb{Z}$-linear combination of sequence operations. The formula may be found in Proposition 2.26, but it is complicated so we begin with some motivation.

Let $f: \bar{m} \rightarrow \bar{k}$ and let $g_{i}: \overline{m_{i}} \rightarrow \overline{j_{i}}$ for $1 \leq i \leq k$. We want to describe the multivariable composition $\langle f\rangle\left(\left\langle g_{1}\right\rangle, \ldots,\left\langle g_{k}\right\rangle\right)$. First observe that

$$
\begin{aligned}
& \langle f\rangle\left(\left\langle g_{1}\right\rangle, \ldots,\left\langle g_{k}\right\rangle\right)\left(x_{1} \otimes \cdots \otimes x_{j_{1}+\cdots+j_{k}}\right) \\
& \quad= \pm\langle f\rangle\left(\left\langle g_{1}\right\rangle\left(x_{1} \otimes \cdots \otimes x_{j_{1}}\right) \otimes \cdots \otimes\left\langle g_{k}\right\rangle\left(x_{j_{1}+\cdots+j_{k-1}+1} \otimes \cdots \otimes x_{j_{1}+\cdots+j_{k}}\right)\right)
\end{aligned}
$$

so we want to evaluate

$$
\langle f\rangle\left(\left\langle g_{1}\right\rangle\left(x_{1} \otimes \cdots \otimes x_{j_{1}}\right) \otimes \cdots \otimes\left\langle g_{k}\right\rangle\left(x_{j_{1}+\cdots+j_{k-1}+1} \otimes \cdots \otimes x_{j_{1}+\cdots+j_{k}}\right)\right)(\sigma) .
$$

To evaluate (2.2), we apply Definition 2.10 twice and this leads to a double sum, with the outer sum indexed by overlapping partitions $\mathcal{A}$ and the inner sum, for each $\mathcal{A}$, indexed by overlapping partitions of $\coprod_{f(j)=i} A_{j}$ for $1 \leq i \leq k$. This suggests the following definition.

Definition 2.20. A double overlapping partition $\mathbb{A}$ of $\{0, \ldots, p\}$ of type $\left(f, m_{1}\right.$, $\left.\ldots, m_{k}\right)$ is an overlapping partition $\mathcal{A}$ of $\{0, \ldots, p\}$ with $m$ pieces together with, for each $1 \leq i \leq k$, an overlapping partition $\mathcal{B}^{i}$ of $\coprod_{f(j)=i} A_{j}$ with $m_{i}$ pieces.

Thus (2.2) is a sum indexed by the double overlapping partitions of type $\left(f, m_{1}\right.$, $\left.\ldots, m_{k}\right)$. Next let us observe that the overlapping partition $\mathcal{A}$ has $m-1$ "overlap points" (see Remark 2.4) and the overlapping partition $\mathcal{B}^{i}$ has $m_{i}-1$ overlap points for each $i$; collecting these together, we get $m-1+\left(\sum m_{i}\right)-k$ points, and using these as overlap points, we get a partition of $\{0, \ldots, p\}$ with $m-k+\sum m_{i}$ pieces: we will call this overlapping partition $\mathcal{C}$ and denote its pieces by $C_{1}, \ldots, C_{m-k+\sum m_{i}}$. 
Now in the expansion of the expression (2.2) each of the $C_{l}$ is associated to one of the cochains $x_{1}, \ldots, x_{j_{1}+\cdots+j_{k}}$; this gives a surjection

$$
h: \overline{m-k+\sum m_{i}} \rightarrow \overline{\sum j_{i}} .
$$

When all the terms in the expansion of (2.2) which give rise to the same $h$ are collected together, it is not hard (if signs are ignored) to see that they add to

$$
\langle h\rangle\left(x_{1} \otimes \cdots \otimes x_{j_{1}+\cdots+j_{k}}\right)(\sigma) .
$$

This shows that the multivariable composition $\langle f\rangle\left(\left\langle g_{1}\right\rangle, \ldots,\left\langle g_{k}\right\rangle\right)$ with which we began is indeed a $\mathbb{Z}$-linear combination of the sequence operations $\langle h\rangle$.

In order to say exactly which $h$ 's occur for a given choice of $f, g_{1}, \ldots, g_{k}$, and in order to specify the signs, and in order to be able to give efficient proofs, we need to reformulate the discussion just given in a more abstract way.

First we give an abstract description of the concept of overlapping partition.

Definition 2.21. Let $S, T$ and $U$ be finite totally ordered sets. A diagram of the form

$$
S \stackrel{\alpha}{\longleftarrow} T \stackrel{\beta}{\longrightarrow} U
$$

is special if

(i) $\alpha$ and $\beta$ are order-preserving epimorphisms, and

(ii) if $q<q^{\prime}$ is an adjacent pair in $T$, then $\alpha(q)<\alpha\left(q^{\prime}\right)$ or $\beta(q)<\beta\left(q^{\prime}\right)$ but not both (this implies that $|T|=|S|+|U|-1$ ).

Lemma 2.22. A special diagram

$$
\{0, \ldots, p\} \stackrel{\alpha}{\longleftarrow} \overline{p+m} \stackrel{\beta}{\longrightarrow} \bar{m}
$$

determines, and is determined by, an overlapping partition $\mathcal{A}$ of $\{0, \ldots, p\}$ with $m$ pieces, with $A_{j}=\alpha\left(\beta^{-1}(j)\right)$.

The (easy) proof is left to the reader.

Our next three definitions are needed to specify the $\langle h\rangle$ 's that arise in evaluating a multivariable composition and the signs that go with them.

Fix $f: \bar{m} \rightarrow \bar{k}$ and nonnegative integers $m_{1}, \ldots, m_{k}$. Let

$$
\chi: \coprod \overline{m_{i}} \rightarrow \bar{k}
$$

be the map which takes $\overline{m_{i}}$ to $i$.

Definition 2.23. A diagram of type $\left(f, m_{1}, \ldots, m_{k}\right)$ is a commutative diagram of the form

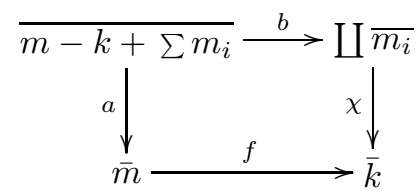

such that $a$ is order preserving and for each $i \in \bar{k}$ the diagram

$$
f^{-1}(i) \stackrel{a}{\longleftarrow} b^{-1}\left(\overline{m_{i}}\right) \stackrel{b}{\longrightarrow} \overline{m_{i}}
$$

is special. 
For example, a double overlapping partition of type $\left(f, m_{1}, \ldots, m_{k}\right)$ gives a diagram of type $\left(f, m_{1}, \ldots, m_{k}\right)$ as follows (see Definition 2.20 and the paragraph which follows it for the notation): we define $a(l)=j$ if $C_{l}$ is contained in the $j$-th piece of the overlapping partition $\mathcal{A}$ and we define $b(l)=n \in \overline{m_{i}}$ if $C_{l}$ is contained in the $n$-th piece of the overlapping partition $\mathcal{B}^{i}$.

Definition 2.24. Given a diagram of type $\left(f, m_{1}, \ldots, m_{k}\right)$ and $i \in \bar{k}$, let $\mathcal{A}^{i}$ be the overlapping partition of $f^{-1}(i)$ with $m_{i}$ pieces given by $\mathcal{A}_{r}^{i}=a\left(b^{-1}(r)\right)$ for $r \in \overline{m_{i}}$.

The diagram is in fact determined by the overlapping partitions $\mathcal{A}^{i}$; it expresses the information contained in these overlapping partitions in a convenient form.

Definition 2.25. Let $f: \bar{m} \rightarrow \bar{k}$ and $g_{i}: \bar{m}_{i} \rightarrow \overline{j_{i}}, 1 \leq i \leq k$, be nondegenerate. For each diagram $D$ of type $\left(f, m_{1}, \ldots, m_{k}\right)$, let $h_{D}$ be the composite

$$
\overline{m-k+\sum m_{i}} \stackrel{b}{\longrightarrow} \amalg \overline{m_{i}} \stackrel{\amalg g_{i}}{\longrightarrow} \amalg \overline{j_{i}} \stackrel{\omega}{\longrightarrow} \overline{\sum j_{i}}
$$

where $\omega$ takes $q \in \overline{j_{i}}$ to $j_{1}+\cdots+j_{i-1}+q$. Let $\eta(D)$ denote

$$
\sum_{1 \leq i<i^{\prime} \leq k}\left(m_{i}-j_{i}\right)\left\|f^{-1}\left(i^{\prime}\right)\right\|+\sum_{i \in \bar{k}} \epsilon\left(g_{i}, \mathcal{A}^{i}\right) .
$$

Finally, we have

Proposition 2.26.

$$
\langle f\rangle\left(\left\langle g_{1}\right\rangle, \ldots,\left\langle g_{k}\right\rangle\right)=\sum_{D}(-1)^{\eta(D)}\left\langle h_{D}\right\rangle
$$

where the sum is taken over all diagrams $D$ of type $\left(f, m_{1}, \ldots, m_{k}\right)$.

A conceptual way to remember the sign is that (in the terminology of Remark 2.11(f)) it comes from permuting the *'s corresponding to $f$ and the $g_{i}$ into the order corresponding to $h$.

We defer the proof of Proposition 2.26 to Section 8

It only remains to prove part (c) of Theorem 2.15. Consider the chain homotopy

$$
s: \mathcal{S}(k)_{q} \rightarrow \mathcal{S}(k)_{q+1}
$$

which places a 1 at the beginning of each sequence; if the sequence already begins with a 1 , then the new sequence is degenerate so $s$ takes it to zero. This chain homotopy has the property that

$$
\partial s+s \partial=\mathrm{id}+\iota r
$$

where $\iota: \mathcal{S}(k-1) \rightarrow \mathcal{S}(k)$ places a 1 at the beginning of each sequence and increases each of the original entries by 1 , and $r: \mathcal{S}(k) \rightarrow \mathcal{S}(k-1)$ takes a sequence to zero unless it begins with a 1 and has no other 1's, in which case $r$ removes the 1 and decreases each of the remaining entries by 1 . Since $r$ is an epimorphism and $\iota$ is a monomorphism, this implies that $\mathcal{S}(k)$ has the same homology as $\mathcal{S}(k-1)$, so the desired result follows by induction.

Remark 2.27. The chain homotopy that was used in this proof is due to Benson [1] page 147]. 


\section{THE CHAIN OPERADS $\mathcal{S}_{n}$}

The normalized singular chain functor $S_{*}$ takes topological operads to chain operads ([14, page 25]). Let $\mathcal{C}_{n}$ be the little $n$-cubes operad ([16],[4]). In this section we define a suboperad $\mathcal{S}_{n}$ of $\mathcal{S}$ which will turn out to be quasi-isomorphic (in the category of chain operads over $\mathbb{Z}$ ) to $S_{*} \mathrm{C}_{n}$.

Definition 3.1. Let $T$ be a finite totally ordered set, let $k \geq 2$, and let $f: T \rightarrow \bar{k}$. We define the complexity of $f$ as follows. If $k$ is 0 or 1 , the complexity is 0 . If $k=2$, let $\sim$ be the equivalence relation on $T$ generated by

$$
a \sim b \text { if } a \text { is adjacent to } b \text { and } f(a)=f(b)
$$

and define the complexity of $f$ to be the number of equivalence classes minus 1 . If $k>2$, define the complexity of $f$ to be the maximum of the complexities of the restrictions $\left.f\right|_{f^{-1}(A)}$ as $A$ ranges over the two-element subsets of $\bar{k}$.

The motivation for this definition is given in Remark 3.6.

Definition 3.2. For each $n \geq 1$ and $k \geq 0$, let $\mathcal{S}_{n}(k)$ be the sub-graded-abeliangroup of $\mathcal{S}(k)$ generated by the nondegenerate $f$ with complexity $\leq n$. Let $\mathcal{S}_{n}$ denote the collection $S_{n}(k), k \geq 0$.

Proposition 3.3. $\mathcal{S}_{n}$ is a sub-chain-operad of $\mathcal{S}$.

For the proof we need a lemma whose proof is left to the reader.

Lemma 3.4. Given a commutative diagram

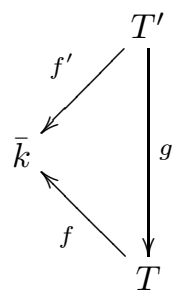

with $g$ ordered, the complexity of $f^{\prime}$ is $\leq$ the complexity of $f$. If $g$ is a surjection, then the complexity of $f^{\prime}$ is equal to the complexity of $f$.

Proof of Proposition 3.3. It is easy to see that the action of $\Sigma_{k}$ on $S_{n}(k)$ preserves complexity. The fact that $\partial$ preserves complexity is immediate from Lemma 3.4 . Now suppose that $f: \bar{m} \rightarrow \bar{k}$ and $g_{i}: \overline{m_{i}} \rightarrow \overline{j_{i}}$ have complexity $\leq n$ and choose a diagram $D$ of type $\left(f, m_{1}, \ldots, m_{k}\right)$ :

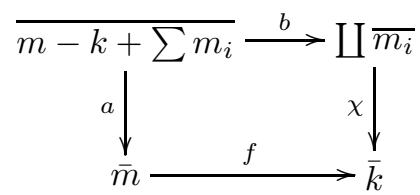

It suffices to show that $h_{D}$ has complexity $\leq n$, and for this we need to show that the restriction of $h_{D}$ to $h_{D}^{-1}(A)$ has complexity $\leq n$ whenever $A$ is a two-element subset of $\overline{\sum j_{i}}$. There are two cases: either $\omega^{-1}(A)$ is contained in some $\overline{j_{i}}$ or not. If $\omega^{-1}(A) \subset \overline{j_{i}}$ for some $i$, then the complexity of $\left.h_{D}\right|_{h_{D}^{-1}(A)}$ is equal to the complexity of $\left.\left(\omega \circ g_{i} \circ b\right)\right|_{h_{D}^{-1}(A)}$, and this is less than or equal to the complexity of $g_{i}$ by Lemma 3.4 (since $b$ is order-preserving on $b^{-1} \overline{m_{i}}$ ). 
If $\omega^{-1}(A)$ is not contained in any $\overline{j_{i}}$, then the complexity of $\left.h_{D}\right|_{h_{D}^{-1}(A)}$ is the same as that of $\left.\chi \circ b\right|_{h_{D}^{-1}(A)}$. But $\chi \circ b=f \circ a$, and the complexity of $\left.f \circ a\right|_{h_{D}^{-1}(A)}$ is less than or equal to the complexity of $f$ by Lemma 3.4 (since $a$ is order-preserving).

Theorem 3.5. $\mathcal{S}_{n}$ is quasi-isomorphic, in the category of chain operads, to $S_{*} \mathrm{e}_{n}$.

This means that there is a sequence

$$
\mathcal{S}_{n} \leftarrow \cdots \rightarrow S_{*} \mathcal{C}_{n}
$$

of chain-operad maps which are quasi-isomorphisms.

Theorem 3.5 will be proved in Section 5 .

Remark 3.6. (Motivation for the definition of complexity) The definition of complexity when $k=0$ is motivated by the fact that $\mathcal{C}_{n}(0)$ is a point for all $n$. The definition when $k=1$ is motivated by the requirement that the unit of $\mathcal{S}$ should be in $\mathcal{S}_{n}$. The definition when $k=2$ is motivated by the fact that $\mathcal{C}_{n}(2)$ is homotopic to $S^{n-1}$; note that $\mathcal{S}_{n}(2)$ is isomorphic to the cellular chain complex of the usual $\mathbb{Z} / 2$-equivariant CW structure on $S^{n-1}$. The definition of complexity when $k>2$ is not as easy to motivate, and it is possible that there are other ways of choosing the complexity filtration so that the analog of Theorem 3.5 is true (note, however, how easy Lemma 3.4 is to prove for the filtration we have given). Our choice was suggested by the filtration used in [19] (the original motivation for the filtration in [19] is that it is the simplest filtration compatible with the "degeneracy maps" from the $k$-th space of the operad to the second space of the operad).

\section{Deligne's Hochschild cohomology conjecture}

In this section we describe a natural action of $\mathcal{S}_{2}$ on the normalized Hochschild cochain complex. Intuitively, the reason there is such an action is that (i) the cup product and brace operations (see [8, 11]) on Hochschild cochains satisfy the same relations as the sequence operations 12 and $12131 \cdots 1$, and (ii) these sequence operations generate $\mathcal{S}(2)$ as an operad.

Let $R$ be an associative ring. Recall that the $p$-th Hochschild cochain group consists of the homomorphisms of abelian groups

$$
x: R^{\otimes p} \rightarrow R
$$

where $R^{\otimes 0}$ is interpreted as $\mathbb{Z}$. The normalized cochain group $C^{p}(R)$ consists of the Hochschild cochains whose composites with each of the maps

$$
R^{\otimes i} \otimes \mathbb{Z} \otimes R^{\otimes(p-i-1)} \stackrel{1 \otimes \iota \otimes 1}{\longrightarrow} R^{\otimes p}
$$

are zero, where $\iota$ is the unit of $R$. The differential $C^{p}(R) \rightarrow C^{p+1}(R)$ is defined by

$$
\begin{aligned}
& (\partial x)\left(r_{1} \otimes \cdots \otimes r_{p+1}\right)=r_{1} \cdot x\left(r_{2} \otimes \cdots \otimes r_{p+1}\right) \\
& \quad+\sum_{0<i<p+1} x\left(r_{1} \otimes \cdots \otimes r_{i} r_{i+1} \otimes \cdots \otimes r_{p+1}\right)+(-1)^{p+1} x\left(r_{1} \otimes \cdots \otimes r_{p}\right) \cdot r_{p+1} .
\end{aligned}
$$

There is also a cup product in $C^{*}(R)$ : if $x \in C^{p}(R)$ and $y \in C^{q}(R)$, then

$$
(x \smile y)\left(r_{1} \otimes \cdots \otimes r_{p+q}\right)=x\left(r_{1} \otimes \cdots \otimes r_{p}\right) \cdot y\left(r_{p+1} \otimes \cdots \otimes r_{p+q}\right) .
$$

Finally, if $x \in C^{k}(R)$ and $x_{i} \in C^{j_{i}}(R)$ for $1 \leq i \leq k$, we define $x\left(x_{1}, \ldots, x_{k}\right) \in$ $C^{j_{1}+\cdots+j_{k}}(R)$ to be the composite

$$
R^{j_{1}+\cdots+j_{k}} \stackrel{x_{1} \otimes \cdots \otimes x_{k}}{\longrightarrow} R^{\otimes k} \stackrel{x}{\longrightarrow} R .
$$


Now assume given a sequence $\mathbf{x}=\left(x_{1}, \ldots, x_{k}\right)$ of normalized Hochschild cochains, a finite totally ordered set $T$, and a map $g: \bar{q} \rightarrow \bar{k}$ of complexity $\leq 2$ with the property that $\left|g^{-1}(i)\right|=\left|x_{i}\right|+1$ for each $i$. By a segment we mean a subset $S$ of $T$ such that $g$ has the same value on the minimum and maximum elements of $S$, and by a maximal segment we mean a segment which is not properly contained in any other segment. Let $S_{1}, \ldots, S_{r}$ denote the maximal segments; the fact that $g$ has complexity $\leq 2$ implies that the sets $S_{j}$ are disjoint. We define a normalized Hochschild cochain $g(\mathbf{x})$ inductively as follows.

(i) If $T$ is empty, then $g(\mathbf{x})$ is the identity cochain in $C^{1}(R)$.

(ii) If $T$ has a single element $t$, then $g(\mathbf{x})$ is $x_{g(t)}$.

(iii) If $r>1$, then $g(\mathbf{x})$ is the cup product $\left.\left.g\right|_{S_{1}}(\mathbf{x}) \smile \cdots \smile g\right|_{S_{r}}(\mathbf{x})$.

(iv) If $r=1$ and $|T|>1$, let $i$ be the value of $g$ at the minimum and maximum values of $T$ and let $t_{1}, \ldots, t_{\left|x_{i}\right|+1}$ be the elements of $g^{-1}(i)$ in increasing order. For each $j<\left|x_{i}\right|+1$ let $A_{j}$ be the set $\left\{t_{j}<t<t_{j+1}\right\}$ (which may be empty) and let

$$
g(\mathbf{x})=x_{i}\left(\left.g\right|_{A_{1}}(\mathbf{x}), \ldots,\left.g\right|_{A_{\left|x_{i}\right|}}(\mathbf{x})\right) .
$$

Now let $f: \bar{m} \rightarrow \bar{k}$ be nondegenerate, and let $\mathbf{x}=\left(x_{1}, \ldots, x_{k}\right)$ be a $k$-tuple in $C^{*}(R)$. We define

$$
\theta_{k}\left(f, x_{1}, \ldots, x_{k}\right)=\sum_{E}(-1)^{\epsilon^{\prime}(f, \mathcal{A})}(f \circ \beta)(\mathbf{x})
$$

where $E$ runs through the special diagrams

$$
\overline{\sum\left|x_{i}\right|+k-m+1} \stackrel{\alpha}{\longleftarrow} \overline{k+\sum\left|x_{i}\right|} \stackrel{\beta}{\longrightarrow} \bar{m}
$$

and $\mathcal{A}$ denotes the overlapping partition of $\overline{k-m+\sum\left|x_{i}\right|}$ with $\mathcal{A}_{j}=\alpha\left(\beta^{-1}(j)\right)$; the sign is given by

$$
\epsilon^{\prime}(f, \mathcal{A})=\epsilon(f, \mathcal{A})+(m-k) \sum\left|x_{i}\right|+\left|\left\{\left(j, j^{\prime}\right): f(j)=f\left(j^{\prime}\right)\right\}\right| .
$$

The term corresponding to $E$ is counted as zero unless, for each $i,\left.\alpha\right|_{\beta^{-1} f^{-1}(i)}$ is a monomorphism and $\left|\beta^{-1} f^{-1}(i)\right|=\left|x_{i}\right|+1$.

Theorem 4.1. The maps $\theta_{k}$ give $C^{*}(R)$ a natural structure of algebra over $\mathcal{S}_{2}$.

The proof is similar to the proofs of Propositions 2.18, 2.19] and 2.26] and is left to the reader.

\section{Proof of Theorem 3.5}

Definition 5.1. For each $k \geq 0$, let $P_{2} \bar{k}$ be the set of subsets of $\bar{k}$ that have two elements.

Definition 5.2. Let $\mathcal{J}(k)$ be the set whose elements are pairs $(b, T)$, where $b$ is a function from $P_{2} \bar{k}$ to the nonnegative integers and $T$ is a total order of $\bar{k}$. We give $\mathcal{J}(k)$ the partial order for which $(a, S) \leq(b, T)$ if $a(\{i, j\}) \leq b(\{i, j\})$ for each $\{i, j\} \in P_{2} \bar{k}$ and $a(\{i, j\})<b(\{i, j\})$ for each $\{i, j\}$ with $i<j$ in the order $S$ but $i>j$ in the order $T$. Let $\mathcal{J}_{n}(k)$ be the subset of pairs $(b, T)$ such that $b\{i, j\}<n$ for each $\{i, j\} \in P_{2} \bar{k}$. The set $\mathcal{J}_{n}(k)$ inherits an order from $\mathcal{J}(k)$. 
The sequence $\mathcal{J}(k)$ is an operad in the category of partially ordered sets with the following structure maps [2]. The right action of $\Sigma_{k}$ on $\mathcal{J}(k)$ is given by

$$
(b, T) \rho=\left(b \circ \rho_{2}, T \rho\right)
$$

where $\rho_{2}: P_{2} \bar{k} \rightarrow P_{2} \bar{k}$ is the function $\rho_{2}(\{i, j\})=\{\rho(i), \rho(j)\}$ and where $i<j$ in the total order $T \rho$ if $\rho(i)<\rho(j)$ in the total order $T$. The operad composition

$$
\mathcal{J}(k) \times \mathcal{J}\left(a_{1}\right) \times \cdots \times \mathcal{J}\left(a_{k}\right) \rightarrow \mathcal{J}\left(\Sigma a_{i}\right)
$$

sends the $(k+1)$-tuple of pairs $\left((b, T) ;\left(b_{1}, T_{1}\right), \ldots,\left(b_{k}, T_{k}\right)\right)$ with $(b, T) \in \mathcal{J}(k)$ and $\left(b_{i}, T_{i}\right) \in \mathcal{J}\left(a_{i}\right)$ to the pair $\left(b\left(b_{1}, \ldots, b_{k}\right), T\left(T_{1}, \ldots, T_{k}\right)\right)$ in $\mathcal{J}(a)$ with $a=\Sigma a_{i}$, where the value of $b\left(b_{1}, \ldots, b_{k}\right)$ at $\{r, s\}$ is $b_{i}(\{r, s\})$ if $\{r, s\} \subset \bar{a}_{i}$ and the value at $\{r, s\}$ is $b(\{i, j\})$ if $r \in \bar{a}_{i}, s \in \bar{a}_{j}$ and $i \neq j$, and where $T\left(T_{1}, \ldots, T_{k}\right)$ is the total order of $\bar{a}=\coprod \bar{a}_{i}$ for which $r<s$ if $\{r, s\} \subset \bar{a}_{i}$ and $r<s$ in the order $T_{i}$ on $\bar{a}_{i}$ or if $r \in \bar{a}_{i}$, $s \in \bar{a}_{j}$ and $i<j$. For each $n$ the sequence of partial orders $\mathcal{J}_{n}(k)$ is a suboperad of the sequence $\mathcal{J}(k)$.

Given a category $\mathcal{J}$, let us write $\mathrm{N} \mathcal{J}$ for the nerve of $\mathcal{J}$ and $C_{*} \mathcal{J}$ for the normalized chains of the simplicial set $\mathrm{N} J$.

Proposition 5.3. The chain operad $C_{*} \mathrm{~J}_{n}(k)$ is quasi-isomorphic to the chain op$\operatorname{erad} S_{*} \mathrm{e}_{n}$.

Proof. Berger has shown 22 that the operad of spaces obtained as the geometric realization of the nerves $\left|\mathrm{NJ}_{n}(k)\right|$ is weakly equivalent to the operad of little $n$ cubes. Now apply the normalized singular chains functor to get a quasi-isomorphism of chain operads between $S_{*}\left|\mathrm{NJ}_{n}(k)\right|$ and $S_{*} \mathrm{C}_{n}$. The natural map $C_{*} \mathcal{J}_{n}(k) \rightarrow$ $S_{*}\left|\mathrm{NJ}_{n}(k)\right|$ is a quasi-isomorphism of chain operads and the proof is complete.

Next, a technique of Berger [2] can be used to prove that the operad $\mathcal{S}_{n}$ is quasi-isomorphic to the operad $C_{*} \mathcal{J}_{n}$.

Definition 5.4. Let $f: \bar{m} \rightarrow \bar{k}$ be a surjective map. Then $\left(b_{f}, T_{f}\right) \in \mathcal{J}(k)$ is the pair where $b_{f}(\{i, j\})$ is one less than the complexity of the restriction of $f$ to a map $f^{-1}(\{i, j\}) \rightarrow\{i, j\}$ and where $i<j$ in the total order $T_{f}$ if the smallest element of $f^{-1}(i)$ is less than the smallest element of $f^{-1}(j)$.

Definition 5.5. For $(b, T) \in \mathcal{J}(k)$, the chain complex $\mathcal{S}(b, T)$ is the subcomplex of $\mathcal{S}(k)$ generated by those surjective functions $f: \bar{m} \rightarrow \bar{k}$ with $\left(b_{f}, T_{f}\right) \leq(b, T)$.

Proposition 5.6. The chain complex $\mathcal{S}(b, T)$ is contractible.

Proof. For $i \in \bar{k}$ let $i_{*} \mathcal{S}(k-1)$ be the subcomplex of $\mathcal{S}(k)$ generated by the sequences that begin with $i$ and have no other occurrences of $i$. In the proof of Theorem 2.15(c) we constructed a chain homotopy $s: \mathcal{S}(k) \rightarrow \mathcal{S}(k)$ that gives a deformation retraction of $\mathcal{S}(k)$ onto $1_{*} \mathcal{S}(k-1)$. For $i \neq 1$ let $\rho_{i}$ be the transposition $(1, i)$ and let $s_{i}=s \circ \rho_{i}$. Then $s_{i}$ is a chain homotopy that gives a deformation retraction of $\mathcal{S}(k)$ onto $i_{*} \mathcal{S}(k)$. If a subcomplex $C$ of $\mathcal{S}(k)$ is invariant under $s_{i}$, then there is a deformation retraction of $C$ onto $C \cap i_{*} \mathcal{S}(k-1)$. In particular, if $i$ is the first element of the total order $T$, then $\mathcal{S}(b, T)$ is invariant under $s_{i}$ and its deformation retract is isomorphic to $\mathcal{S}\left(\delta_{i}^{*} b, \delta_{i}^{*} T\right)$ where $\delta_{i}: \overline{k-1} \rightarrow \bar{k}$ is the unique order-preserving monomorphism that does not have $i$ in its image, $\delta_{i}^{*} b$ is the restriction of $b$ to $P_{2} \overline{k-1}$ and $\delta_{i}^{*} T$ is the pullback of $T$ to a total order of $\overline{k-1}$. It then follows by induction that $\mathcal{S}(b, T)$ is contractible. 
Let $\mathbf{C}$ denote the category of nonnegatively graded chain complexes of abelian groups.

Definition 5.7. Let $D_{n}(k): \mathcal{J}_{n}(k) \rightarrow \mathbf{C}$ be the diagram of chain complexes given by

$$
D_{n}(k)(b, T)=\mathcal{S}(b, T)
$$

for $(b, T) \in \mathcal{J}_{n}(k)$.

A function $f: \bar{m} \rightarrow \bar{k}$ is in $\mathcal{S}(b, T)$ if and only $\left(b_{f}, T_{f}\right) \leq(b, T)$. It follows that the natural map

$$
\operatorname{colim}_{\mathcal{J}_{n}(k)} D_{n}(k) \rightarrow \mathcal{S}_{n}(k)
$$

is an isomorphism. We wish to study the homotopy colimit of $D_{n}(k)$ and so we recall (from [10] Section 20.1]) the definition of the homotopy colimit in the category of chain complexes. For a partially ordered set $\mathcal{J}$, let $a / \mathcal{J}$ denote the suborder of all elements of $b \in \mathcal{J}$ such that $a \geq b$. Notice that $C_{*}(-/ \mathcal{J})$ is a contravariant functor from $\mathcal{J}$ to $\mathbf{C}$

Definition 5.8. Let $\mathcal{J}$ be a partially ordered set and let $D: \mathcal{J} \rightarrow \mathcal{C}$ be a diagram of chain complexes. The homotopy colimit of $D$ is the coequalizer in $\mathbf{C}$

$$
\bigoplus_{a<b} C_{*}(b / \mathcal{J}) \otimes D a \rightrightarrows \bigoplus_{a} C_{*}(a / \mathcal{J}) \otimes D a \rightarrow \operatorname{hocolim} D .
$$

As a special case, if $D$ is the constant functor with value $\mathbb{Z}$, then $\operatorname{hocolim} D=$ $C_{*} \mathcal{J}$

Proposition 5.9. Let $\mathcal{J}$ be a partially ordered set and let $D: \mathcal{J} \rightarrow \mathcal{C}$ be a diagram of chain complexes. If the map $\operatorname{colim}_{a<b} D a \rightarrow D b$ is a monomorphism for each $b \in \mathcal{J}$, then the natural map hocolim $D \rightarrow \operatorname{colim} D$ is a quasi-isomorphism.

Proof. This is a general property of homotopy colimits; see [10, Theorem 20.9.1]. Give $\mathcal{C}$, the category of chain complexes, the model structure for which the cofibrations are the monomorphisms and the weak equivalences are the quasi-isomorphisms. Then $D: \mathcal{J} \rightarrow \mathcal{C}$ is a Reedy cofibrant diagram and hocolim $D \rightarrow \operatorname{colim} D$ is a quasiisomorphism.

For $\rho \in \Sigma_{k}$, the action of $\rho$ on $\mathcal{S}(k)$ restricts to a natural map

$$
\rho^{*}: \mathcal{S}(b, T) \rightarrow \mathcal{S}((b, T) \rho) .
$$

This is a natural transformation of functors on $\mathcal{J}_{n}(k)$ and induces a map of homotopy colimits

$$
\rho^{*}: \operatorname{hocolim} D_{n}(k) \rightarrow \operatorname{hocolim} D_{n}(k) .
$$

Also, the operad composition of $\mathcal{S}$ induces a natural transformation

$$
\mathcal{S}(b, T) \otimes \bigotimes_{i} \mathcal{S}\left(b_{i}, T_{i}\right) \rightarrow \mathcal{S}\left(b\left(b_{1}, \ldots, b_{k}\right), T\left(T_{1}, \ldots, T_{k}\right)\right)
$$

and this induces a map

$$
\operatorname{hocolim} D_{n}(k) \otimes \bigotimes_{i} \operatorname{hocolim} D_{n}\left(a_{i}\right) \rightarrow \operatorname{hocolim} D_{n}\left(\Sigma a_{i}\right) .
$$

Chasing diagrams gives:

Proposition 5.10. For each $n \geq 1$ the sequence of chain complexes hocolim $D_{n}(k)$ is an operad. 
Now we can complete the proof of Theorem 3.5. We have a diagram of chain operads

$$
C_{*} \mathcal{J}_{n}(k)=\operatorname{hocolim}_{\mathcal{J}_{n}(k)} \mathbb{Z} \leftarrow \operatorname{hocolim}_{\mathcal{J}_{n}(k)} \mathcal{S}(b, T) \rightarrow \operatorname{colim}_{\mathcal{J}_{n}(k)} \mathcal{S}(b, T)=\mathcal{S}_{n}(k) .
$$

Since the chain complexes $\mathcal{S}(b, T)$ are contractible, the left-hand map is a quasiisomorphism of chain operads. Since the diagrams $D_{n}(k)$ satisfy the condition in Proposition 5.9, the right-hand map is also a quasi-isomorphism of chain operads.

\section{Proof of Proposition 2.19}

Recall the homomorphism

$$
[f]: \mathcal{S}_{*} X \rightarrow\left(S_{*} X\right)^{\otimes k}
$$

from Definition 2.10(a). To prove Proposition 2.19, it suffices to show

$$
\rho \circ[f]=(-1)^{\zeta(f, \rho)}[\rho \circ f]
$$

Recall that when $A$ is a set, we are using $\|A\|$ to denote the cardinality of $A$ minus 1 .

Let us fix an $i$ with $1 \leq i \leq k$. It suffices to verify (6.1) when $\rho$ is the transposition permuting $i$ and $i+1$. We need to show that

$$
\epsilon(f, \mathcal{A})+\left\|\coprod_{f(j)=i} A_{j}\right\|\left\|\coprod_{f(j)=i+1} A_{j}\right\| \equiv \zeta(f, \rho)+\epsilon(\rho \circ f, \mathcal{A}) \quad \bmod 2 .
$$

Unwinding the definitions, the left-hand side of (6.2) becomes

$$
\begin{aligned}
\sum_{\substack{j<j^{\prime} \\
f(j)>f\left(j^{\prime}\right)}}\left\|A_{j}\right\|\left\|A_{j^{\prime}}\right\|+\sum_{j=1}^{m}\left\|A_{j}\right\|\left(\tau_{f}(j)-f(j)\right)+ & \\
& \left(\left\|f^{-1}(i)\right\|+\sum_{j \in f^{-1}(i)}\left\|A_{j}\right\|\right)\left(\left\|f^{-1}(i+1)\right\|+\sum_{j \in f^{-1}(i+1)}\left\|A_{j}\right\|\right)
\end{aligned}
$$

and the right-hand side becomes

$$
\left\|f^{-1}(i)\right\|\left\|f^{-1}(i+1)\right\|+\sum_{\substack{j<j^{\prime} \\ \rho f(j)>\rho f\left(j^{\prime}\right)}}\left\|A_{j}\right\|\left\|A_{j^{\prime}}\right\|+\sum_{j=1}^{m}\left\|A_{j}\right\|\left(\tau_{\rho f}(j)-\rho f(j)\right) .
$$

The fact that the left- and right-hand sides of (6.2) are congruent mod 2 follows easily from the fact that

$$
\tau_{\rho \circ f}(j)-(\rho \circ f)(j)= \begin{cases}\tau_{f}(j)+\left\|f^{-1}(i+1)\right\| & \text { if } f(j)=i \\ \tau_{f}(j)-\left\|f^{-1}(i)\right\| & \text { if } f(j)=i+1 \\ \tau_{f}(j)-f(j) & \text { otherwise }\end{cases}
$$

\section{Proof of Proposition 2.18}

For this proof it will be convenient to use special diagrams instead of overlapping partitions (see Definition 2.21 and Lemma 2.22) so we begin by reformulating Definitions 2.9 and 2.10 in this language. 
Definition 7.1. Given a special diagram

$$
E: \quad\{0, \ldots, p\} \stackrel{\alpha}{\longleftarrow} \overline{p+m} \stackrel{\beta}{\longrightarrow} \bar{m}
$$

and a nondegenerate $f: \bar{m} \rightarrow \bar{k}$, let

$$
\epsilon(f, E)=\sum_{\substack{j<j^{\prime} \\ f(j)>f\left(j^{\prime}\right)}}\left\|\beta^{-1}(j)\right\|\left\|\beta^{-1}\left(j^{\prime}\right)\right\|+\sum_{j=1}^{m}\left\|\beta^{-1}(j)\right\|\left(\tau_{f}(j)-f(j)\right) .
$$

Using Lemma 2.22, we may rewrite Definition 2.10 as

$$
\sigma[f]=\sum_{E}(-1)^{\epsilon(f, E)} \bigotimes_{i=1}^{k} \sigma\left(\alpha\left(\beta^{-1} f^{-1}(i)\right)\right)
$$

where the sum is taken over all special diagrams

$$
E: \quad\{0, \ldots, p\} \stackrel{\alpha}{\longleftarrow} \overline{p+m} \stackrel{\beta}{\longrightarrow} \bar{m}
$$

and the term corresponding to $E$ is counted as zero unless $\alpha$ is a monomorphism on $\beta^{-1} f^{-1}(i)$ for each $i$.

Now fix $f: \bar{m} \rightarrow \bar{k}$ and $\sigma: \Delta^{p} \rightarrow X$. To prove Proposition 2.18, it suffices to show

$$
\partial((\sigma)[f])=(\partial \sigma)[f]+(-1)^{p} \sum_{j=1}^{m}(-1)^{\tau_{f}(j)-f(j)} \sigma\left[f_{\bar{m}-\{j\}}\right] .
$$

First we analyze the left side of equation (7.2). By equation (7.1) it is equal to

$$
\partial\left(\sum_{E}(-1)^{\epsilon(f, E)} \bigotimes_{i=1}^{k} \sigma\left(\alpha\left(\beta^{-1} f^{-1}(i)\right)\right)\right) .
$$

For each pair $(E, q)$ with $q \in \overline{p+m}$ let us write $E_{q}$ for the diagram

$$
\alpha(\overline{p+m}-\{q\}) \stackrel{\alpha_{q}}{\longleftarrow} \overline{p+m}-\{q\} \stackrel{\beta_{q}}{\longrightarrow} \beta(\overline{p+m}-\{q\})
$$

where $\alpha_{q}$ and $\beta_{q}$ are the restrictions of $\alpha$ and $\beta$ (we are not claiming that all of the diagrams $E_{q}$ obtained in this way are special). Let $f_{q}$ be the restriction of $f$ to $\beta(\overline{p+m}-\{q\})$. Then expression (7.3) can be rewritten as

$$
\sum_{E, q}(-1)^{\epsilon(f, E)} \bigotimes_{i=1}^{k}(-1)^{\kappa(f, E, q)} \sigma\left(\alpha_{q}\left(\beta_{q}^{-1} f_{q}^{-1}(i)\right)\right)
$$

where

$$
\kappa(f, E, q)=\sum_{i<f(\beta(q))}\left\|\beta^{-1} f^{-1}(i)\right\|+\left|\left\{q^{\prime}<q: f\left(\beta\left(q^{\prime}\right)\right)=f(\beta(q))\right\}\right| .
$$

Next let us divide the pairs $(E, q)$ into four types:

- $(E, q)$ is of type I if $1<q<p+m$ and $\alpha(q-1)<\alpha(q)=\alpha(q+1)$.

- $(E, q)$ is of type II if $1<q<p+m$ and $\alpha(q-1)=\alpha(q)<\alpha(q+1)$.

- (E,q) is of type III if $1<q<p+m$ and $\alpha(q-1)<\alpha(q)<\alpha(q+1)$, or if $q=1$ and $\alpha(1)<\alpha(2)$, or if $q=p+m$ and $\alpha(p+m-1)<\alpha(p+m)$.

- (E,q) is of type IV if $1<q<p+m$ and $\alpha(q-1)=\alpha(q)=\alpha(q+1)$, or if $q=1$ and $\alpha(1)=\alpha(2)$, or if $q=p+m$ and $\alpha(p+m-1)=\alpha(p+m)$. 
To complete the proof of Proposition 2.18, it suffices to show

Lemma 7.2. In expression (7.4),

(a) the terms of type I cancel the terms of type II,

(b) the terms of type III add up to $(\partial \sigma)[f]$,

(c) the terms of type IV add up to $(-1)^{p} \sum_{j=1}^{m}(-1)^{\tau_{f}(j)-f(j)} \sigma\left[f_{\bar{m}-\{j\}}\right]$.

Proof of Lemma 7.2. First we simplify equation (7.5). We have

$$
\begin{aligned}
\kappa(f, E, q)= & q-1-\sum_{\substack{j<\beta(q) \\
f(j) \neq f(\beta(q))}}\left|\beta^{-1}(j)\right|+\sum_{f(j)<f(\beta(q))}\left|\beta^{-1}(j)\right|-(f(\beta(q))-1) \\
= & q-f(\beta(q))-\sum_{\substack{j<\beta(q) \\
f(j)>f(\beta(q))}}\left|\beta^{-1}(j)\right|+\sum_{\substack{j>\beta(q) \\
f(j)<f(\beta(q))}}\left|\beta^{-1}(j)\right| \\
= & q-f(\beta(q))-\sum_{\substack{j<\beta(q) \\
f(j)>f(\beta(q))}}|| \beta^{-1}(j)||+\sum_{\substack{j>\beta(q) \\
f(j)<f(\beta(q))}}\left|\beta^{-1}(j)\right| \mid \\
& -|\{j<\beta(q): f(j)>f(\beta(q))\}|+|\{j>\beta(q): f(j)<f(\beta(q))\}| .
\end{aligned}
$$

Using the fact that

$$
\tau_{f}(\beta(q))=|\{j \leq \beta(q): f(j) \leq f(\beta(q))\}|+|\{j>\beta(q): f(j)<f(\beta(q))\}|
$$

and the fact that

$$
\alpha(q)=q-\beta(q)
$$

the last expression simplifies to

$$
\kappa(f, E, q)=\alpha(q)+\tau_{f}(\beta(q))-f(\beta(q))-\sum_{\substack{j<\beta(q) \\ f(j)>f(\beta(q))}}\left\|\beta^{-1}(j)\right\|+\sum_{\substack{j>\beta(q) \\ f(j)<f(\beta(q))}}\left\|\beta^{-1}(j)\right\| .
$$

From equation (7.7) it is easy to see that if $(E, q)$ is of type I, II or III, we have

$$
\epsilon(f, E)+\kappa(f, E, q) \equiv \alpha(q)+\epsilon\left(f, E_{q}\right) \bmod 2 .
$$

Now suppose $E$ is of type I. Define

$$
E^{\prime}: \quad\{0, \ldots, p\} \stackrel{\alpha^{\prime}}{\longleftarrow} \overline{p+m} \stackrel{\beta^{\prime}}{\longrightarrow} \bar{m}
$$

by letting

$$
\alpha^{\prime}(r)= \begin{cases}\alpha(r) & \text { if } r \neq q \\ \alpha(q)-1 & \text { if } r=q\end{cases}
$$

and

$$
\beta^{\prime}(r)= \begin{cases}\beta(r) & \text { if } r \neq q, \\ \beta(q)+1 & \text { if } r=q .\end{cases}
$$

Then $E^{\prime}$ is of type II, and $E_{q}^{\prime}$ is the same diagram as $E_{q}$, but $\alpha^{\prime}(q)=\alpha(q)-1$. Now equation (7.8) implies that the terms in the expression (7.4) corresponding to $E$ and $E^{\prime}$ cancel, which completes the proof of part (a).

For part (b), let $E$ be a diagram of type III. In this case

$$
\alpha(\overline{p+m}-\{q\})=\{0, \ldots, p\}-\{\alpha(q)\},
$$


so we want to show

$$
\begin{aligned}
p(m-k)+\epsilon(f, E)+\kappa(f, E, q) \equiv & \\
& (m-k)+(p-1)(m-k)+\alpha(q)+\epsilon\left(f, E_{q}\right) \quad \bmod 2
\end{aligned}
$$

which is immediate from equation (7.8).

For part (c), let $E$ be a diagram of type IV. In this case

$$
\beta(\overline{p+m}-\{q\})=\bar{m}-\{\beta(q)\}
$$

so we need to show

$$
\epsilon(f, E)+\kappa(f, E, q) \equiv p+\tau_{f}(\beta(q))-f(\beta(q))+\epsilon\left(f_{q}, E_{q}\right) \bmod 2 .
$$

Expanding the definitions and making the obvious cancellations, equation (7.9) reduces to

$$
\begin{aligned}
\alpha(q)-\sum_{\substack{j<\beta(q) \\
f(j)>f(\beta(q))}}\left\|\beta^{-1}(j)\right\|+\sum_{\substack{j>\beta(q) \\
f(j)<f(\beta(q))}}\left\|\beta^{-1}(j)\right\| & +p+\sum_{j=1}^{m}\left\|\beta^{-1}(j)\right\|\left(\tau_{f}(j)-f(j)\right) \\
& \equiv \sum_{j \neq q}\left\|\beta^{-1}(j)\right\|\left(\tau_{f}^{\prime}(j)-f(j)\right) .
\end{aligned}
$$

Here

$$
\tau_{f}^{\prime}(j)= \begin{cases}\tau_{f}(j)-1 & \text { if } j>\beta(q) \text { and } f(j)=f(\beta(q)), \text { or if } f(j)>f(\beta(q)) \\ \tau_{f}(j) & \text { otherwise }\end{cases}
$$

so equation (7.10) reduces to

$$
\alpha(q)+p \equiv \sum_{j>\beta(q)}\left\|\beta^{-1}(j)\right\| \bmod 2
$$

and this follows from equation (7.6) and the fact that

$$
\sum_{j>\beta(q)}\left\|\beta^{-1}(j)\right\|=\sum_{j>\beta(q)}\left|\beta^{-1}(j)\right|-(m-\beta(q))=p+m-q-(m-\beta(q)) .
$$

\section{Proof of Proposition 2.26}

Fix nondegenerate maps $f: \bar{m} \rightarrow \bar{k}$ and $g_{i}: \bar{m}_{i} \rightarrow \bar{j}_{i}$ for $1 \leq i \leq k$. Also fix $\sigma: \Delta^{p} \rightarrow X$. We need to show

$$
\begin{aligned}
\langle f\rangle\left(\left\langle g_{1}\right\rangle, \ldots,\left\langle g_{k}\right\rangle\right)\left(x_{1} \otimes \cdots \otimes\right. & \left.x_{j_{1}+\cdots+j_{k}}\right)(\sigma) \\
& =\sum_{D}(-1)^{\eta(D)}\left\langle h_{D}\right\rangle\left(x_{1} \otimes \cdots \otimes x_{j_{1}+\cdots+j_{k}}\right)(\sigma)
\end{aligned}
$$

where $D$ runs through the diagrams of type $\left(f, m_{1}, \ldots, m_{k}\right)$.

For the proof we will work with special diagrams instead of overlapping partitions (see Definition 2.21 and Lemma 2.22). 
First we apply equation (7.1) to expand the right-hand side of (8.1):

$$
\begin{aligned}
& \text { (8.2) } \sum_{D}(-1)^{\eta(D)}\left\langle h_{D}\right\rangle\left(x_{1} \otimes \cdots \otimes x_{j_{1}+\cdots+j_{k}}\right)(\sigma) \\
& =\left(x_{1} \otimes \cdots \otimes x_{j_{1}+\cdots+j_{k}}\right)\left(\sum_{D}(-1)^{\eta(D)} \sum_{F}(-1)^{\left|h_{D}\right|+\epsilon(h, F)} \bigotimes_{q=1}^{\sum_{j}} \sigma\left(\phi\left(\psi^{-1} h_{D}^{-1}(q)\right)\right)\right)
\end{aligned}
$$

where $F$ runs through the special diagrams of the form

$$
\{0, \ldots, p\} \stackrel{\phi}{\longleftarrow} \overline{p+m-k+\sum m_{i}} \stackrel{\psi}{\longrightarrow} \overline{m-k+\sum m_{i}} .
$$

Next we apply equation (7.1) twice to expand the left-hand side of (8.1):

$$
\begin{aligned}
\langle f\rangle\left(\left\langle g_{1}\right\rangle, \ldots,\left\langle g_{k}\right\rangle\right)\left(x_{1} \otimes \cdots \otimes x_{j_{1}+\cdots+j_{k}}\right)(\sigma) & \\
= & \left(x_{1} \otimes \cdots \otimes x_{j_{1}+\cdots+j_{k}}\right)\left((-1)^{|f|+\sum\left|g_{i}\right|} \sum_{E}(-1)^{\epsilon(f, E)}\right. \\
& \bigotimes_{i=1}^{k}\left(\sum_{E_{i}}(-1)^{\sum_{i>i^{\prime}}\left(m_{i^{\prime}}-j_{i^{\prime}}\right)|| \beta^{-1} f^{-1}(i)||+\epsilon\left(g_{i}, E_{i}\right)} \bigotimes_{r=1}^{j_{i}} \sigma\left(\alpha_{i}\left(\beta_{i}^{-1} g_{i}^{-1}(r)\right)\right)\right) .
\end{aligned}
$$

Here $E$ runs through the special diagrams

$$
\{0, \ldots, p\} \stackrel{\alpha}{\longleftarrow} \overline{p+m} \stackrel{\beta}{\longrightarrow} \bar{m}
$$

which satisfy

$$
\alpha \text { is a monomorphism on } \beta^{-1} f^{-1}(i) \text { for each } i \in \bar{k}
$$

(terms which do not satisfy this condition will be zero because they contain a degenerate simplex) and, for each choice of $E$ and $i, E_{i}$ runs through the special diagrams

$$
\beta^{-1} f^{-1}(i) \stackrel{\alpha_{i}}{\longleftarrow} \overline{\left\|\beta^{-1} f^{-1}(i)\right\|+m_{i}} \stackrel{\beta_{i}}{\longrightarrow} \bar{m}_{i}
$$

which satisfy

$$
\alpha_{i} \text { is a monomorphism on } \beta_{i}^{-1} g_{i}^{-1}(r) \text { for each } r \in \overline{j_{i}} \text {. }
$$

In order to compare equations (8.2) and (8.3), we must first find the relationship between the indexing sets for the double sums.

Let $\mathbf{S}_{1}$ be the set of all $(k+1)$-tuples $\left(E, E_{1}, \ldots, E_{k}\right)$, where $E$ is of the form (8.4) and satisfies (8.5) and $E_{i}$ is of the form (8.6) and satisfies (8.7) $\left(\mathbf{S}_{1}\right.$ is the indexing set for the double sum in equation (8.2)). Let $\mathbf{S}_{2}$ be the set of all pairs $(D, F)$, where $D$ is a diagram

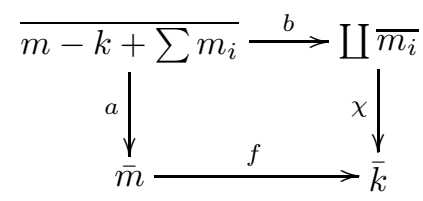


of type $\left(f, m_{1}, \ldots, m_{k}\right)$ and $F$ is a special diagram of the form

$$
\{0, \ldots, p\} \stackrel{\phi}{\longleftarrow} \overline{p+m-k+\sum m_{i}} \stackrel{\psi}{\longrightarrow} \overline{m-k+\sum m_{i}}
$$

satisfying

$$
\phi \text { is a monomorphism on } \psi^{-1} b^{-1} g_{i}^{-1}(r) \text { for each } r \in \overline{j_{i}}
$$

$\left(\mathbf{S}_{2}\right.$ is the indexing set for the double sum in equation (8.3); terms which do not satisfy condition (8.8) are zero because they contain a degenerate simplex). We can define a function

$$
\Upsilon: \mathbf{S}_{2} \rightarrow \mathbf{S}_{1}
$$

as follows. Given a pair $(D, F)$ in $\mathbf{S}_{2}$, let $T$ denote the quotient of $\overline{p+m-k+\sum m_{i}}$ by the equivalence relation $\sim$ generated by

$$
s \sim s^{\prime} \text { if } s \text { and } s^{\prime} \text { are adjacent, } \phi(s)=\phi\left(s^{\prime}\right) \text { and } a \circ \psi(s)=a \circ \psi\left(s^{\prime}\right) .
$$

Then the diagram

$$
\{0, \ldots, p\} \longleftarrow T \longrightarrow \bar{m},
$$

where the left arrow is induced by $\phi$ and the right by $b \circ \psi$, is special. Using the fact that there is a unique ordered bijection $T \rightarrow \overline{p+m}$, we obtain a special diagram

$$
E: \quad\{0, \ldots, p\} \stackrel{\alpha}{\longleftarrow} \overline{p+m} \stackrel{\beta}{\longrightarrow} \bar{m}
$$

and this diagram satisfies (8.5). Note that for this diagram we have

$$
\alpha\left(\beta^{-1} f^{-1}(i)\right)=\phi\left(\psi^{-1} b^{-1}\left(\overline{m_{i}}\right)\right) .
$$

Next let $T_{i}=\psi^{-1} b^{-1}\left(\overline{m_{i}}\right)$. Using condition (8.8) we see that the diagram

$$
\phi\left(\psi^{-1} b^{-1} \overline{m_{i}}\right) \stackrel{\phi}{\longleftarrow} T_{i} \stackrel{b \circ \psi}{\longrightarrow} \overline{m_{i}}
$$

is special, and using equation (8.9) we see that

$$
\left|T_{i}\right|=\left\|\beta^{-1} f^{-1}(i)\right\|+m_{i}
$$

so that we get a special diagram

$$
E_{i}: \quad \beta^{-1} f^{-1}(i) \stackrel{\alpha_{i}}{\llbracket} \overline{\left\|\beta^{-1} f^{-1}(i)\right\|+m_{i}} \stackrel{\beta_{i}}{\longrightarrow} \bar{m}_{i}
$$

and this diagram satisfies (8.7). Finally, we define

$$
\Upsilon(D, F)=\left(E, E_{1}, \ldots, E_{k}\right) .
$$

Lemma 8.1. $\Upsilon$ is a bijection.

We defer the proof to the end of this section.

Inspection of equations (8.2) and (8.3) and Definition[2.25]shows that Proposition 2.26 will follow from:

Lemma 8.2. If $\Upsilon(D, F)=\left(E, E_{1}, \ldots, E_{k}\right)$, then

$$
\epsilon(f, E)+\sum_{i>i^{\prime}}\left(m_{i^{\prime}}-j_{i^{\prime}}\right)\left\|\beta^{-1} f^{-1}(i)\right\|+\sum_{i} \epsilon\left(g_{i}, E_{i}\right) \equiv \eta(D)+\epsilon(h, F) \quad \bmod 2 .
$$


Proof of Lemma 8.2. After substituting the definitions of $\epsilon(f, E), \epsilon\left(g_{i}, E_{i}\right)$, and $\epsilon(h, F)$ (see Definition 7.1) and the definition of $\eta(D)$ (see Definition 2.25), equation (8.10) becomes

$$
\begin{aligned}
& \sum_{\substack{j<j^{\prime} \\
f(j)>f\left(j^{\prime}\right)}}\left\|\beta^{-1}(j)\right\|\left\|\beta^{-1}\left(j^{\prime}\right)\right\|+\sum_{j=1}^{m}\left\|\beta^{-1}(j)\right\|\left(\tau_{f}(j)-f(j)\right) \\
& +\sum_{i>i^{\prime}}\left(m_{i^{\prime}}-j_{i^{\prime}}\right)\left\|\beta^{-1} f^{-1}(i)\right\|+\sum_{i} \sum_{\substack{r<r^{\prime} \text { in } \overline{m_{i}} \\
g_{i}(r)>g_{i}\left(r^{\prime}\right)}}\left\|\beta_{i}^{-1}(r)\right\|\left\|\beta_{i}^{-1}\left(r^{\prime}\right)\right\| \\
& +\sum_{i} \sum_{r=1}^{m_{i}}\left\|\beta_{i}^{-1}(r)\right\|\left(\tau_{g_{i}}(r)-g_{i}(r)\right) \equiv \sum_{i<i^{\prime}}\left(m_{i}-j_{i}\right)\left\|f^{-1}\left(i^{\prime}\right)\right\| \\
& +\sum_{\substack{r<r^{\prime} \text { in } \overline{m_{i}} \\
g_{i}(r)>g_{i}\left(r^{\prime}\right)}}\left\|b^{-1}(r)\right\|\left\|b^{-1}\left(r^{\prime}\right)\right\|+\sum_{i} \sum_{r=1}^{m_{i}}\left\|b^{-1}(r)\right\|\left(\tau_{g_{i}}(r)-g_{i}(r)\right) \\
& +\sum_{\substack{s<s^{\prime} \\
h(s)>h\left(s^{\prime}\right)}}\left\|\psi^{-1}(s)\right\|\left\|\psi^{-1}\left(s^{\prime}\right)\right\|+\sum_{s=1}^{m-k+\sum m_{i}}\left\|\psi^{-1}(s)\right\|\left(\tau_{h}(s)-h(s)\right) .
\end{aligned}
$$

Next we substitute the equations

$$
\begin{gathered}
\left\|\beta^{-1} f^{-1}(i)\right\|=\left\|f^{-1}(i)\right\|+\sum_{f(j)=i}\left\|\beta^{-1}(j)\right\|, \\
\left\|\beta^{-1}(j)\right\|=\sum_{a(s)=j}\left\|\psi^{-1}(j)\right\|,
\end{gathered}
$$

and

$$
\left\|\beta_{i}^{-1}(r)\right\|=\left\|b^{-1}(r)\right\|+\sum_{b(s)=r}\left\|\psi^{-1}(s)\right\|
$$

and cancel; this reduces equation (8.11) to

$$
\begin{gathered}
\sum_{\substack{a(s)<a\left(s^{\prime}\right) \\
(a(s))>f\left(a\left(s^{\prime}\right)\right)}}\left\|\psi^{-1}(s)\right\|\left\|\psi^{-1}\left(s^{\prime}\right)\right\|+\sum_{s=1}^{m-k+\sum m_{i}}\left\|\psi^{-1}(s)\right\|\left(\tau_{f}(a(s))-f(a(s))\right) \\
+\sum_{f(a(s))>i^{\prime}}\left(m_{i^{\prime}}-j_{i^{\prime}}\right)\left\|\psi^{-1}(s)\right\|+\sum_{\substack{b(s)<b\left(s^{\prime}\right) \text { in } \overline{m_{i}} \\
g_{i}(b(s))>g_{i}\left(b\left(s^{\prime}\right)\right)}}\left\|\psi^{-1}(s)\right\|\left\|\psi^{-1}\left(s^{\prime}\right)\right\| \\
+\sum_{\substack{r<b(s) \text { in } \overline{m_{i}} \\
g_{i}(r)>g_{i}(b(s))}}\left\|b^{-1}(r)\right\|\left\|\psi^{-1}(s)\right\|+\sum_{\substack{r>b(s) \text { in } \overline{m_{i}} \\
g_{i}(r)<g_{i}(b(s))}}\left\|b^{-1}(r)\right\|\left\|\psi^{-1}(s)\right\| \\
+\sum_{s=1}^{m-k+\sum m_{i}}\left\|\psi^{-1}(s)\right\|\left(\tau_{g_{i}}(b(s))-g_{i}(b(s))\right)
\end{gathered}
$$




$$
\equiv \sum_{\substack{s<s^{\prime} \\ h(s)>h\left(s^{\prime}\right)}}\left\|\psi^{-1}(s)\right\|\left\|\psi^{-1}\left(s^{\prime}\right)\right\|+\sum_{s=1}^{m-k+\sum m_{i}}\left\|\psi^{-1}(s)\right\|\left(\tau_{h}(s)-h(s)\right) .
$$

Now the first and fourth terms on the left side of (8.12) cancel the second term on the right; if we collect the coefficients of $\left\|\psi^{-1}(s)\right\|$ in all uncanceled terms, it remains to verify, for each $s$, the following equation (where $i$ denotes $f(a(s))$ )

$$
\begin{aligned}
\tau_{f}(a(s))-f(a(s))+ & \sum_{f(a(s))>i^{\prime}}\left(m_{i^{\prime}}-j_{i^{\prime}}\right)+\sum_{\substack{r<b(s) \text { in } \overline{m_{i}} \\
g_{i}(r)>g_{i}(b(s))}}\left\|b^{-1}(r)\right\| \\
& +\sum_{\substack{r>b(s) \text { in } \overline{m_{i}} \\
g_{i}(r)<g_{i}(b(s))}}\left\|b^{-1}(r)\right\|+\left(\tau_{g_{i}}(b(s))-g_{i}(b(s))\right) \equiv\left(\tau_{h}(s)-h(s)\right)
\end{aligned}
$$

and this follows from Definition 2.7 and the equations

$$
\begin{gathered}
h(s)=g_{i}(s)+\sum_{i^{\prime}<i} j_{i}, \\
\tau_{h}(s)=\sum_{i^{\prime}<i}\left|b^{-1}\left(\overline{m_{i^{\prime}}}\right)\right|+\mid\left\{s^{\prime} \in b^{-1}\left(\overline{m_{i}}\right): g_{i}\left(s^{\prime}\right)<g_{i}(s) \text { or } g_{i}\left(s^{\prime}\right)=g_{i}(s) \text { and } s^{\prime} \leq s\right\} \mid, \\
\left|b^{-1}\left(\overline{m_{i^{\prime}}}\right)\right|=m_{i^{\prime}}+\left\|f^{-1}\left(i^{\prime}\right)\right\|
\end{gathered}
$$

(this equation follows from the fact that $E_{i^{\prime}}$ is special) and

$$
\left|\left\{s^{\prime} \in b^{-1}\left(\overline{m_{i}}\right): s^{\prime} \leq s\right\}\right|=\left|\left\{j \in f^{-1}(i): j \leq a(s)\right\}\right|+\left|\left\{r \in \overline{m_{i}}: r \leq b(s)\right\}\right|-1
$$

(this equation follows from the fact that $E_{i}$ is special).

It remains to give the proof of Lemma 8.1 We will define a map $\Phi: \mathbf{S}_{1} \rightarrow \mathbf{S}_{2}$ which is inverse to $\Upsilon$. Let $E$ be a diagram of the form (8.4) and for each $i$ let $E_{i}$ be a diagram of the form (8.6). Suppose that $\left(E, E_{1}, \ldots, E_{k}\right)$ is in $\mathbf{S}_{1}$. Let $T_{i}=\overline{\left\|\beta^{-1} f^{-1}(i)\right\|+m_{i}}$, let

$$
c: \coprod T_{i} \rightarrow \bar{m}
$$

be the map which restricts on $T_{i}$ to $\alpha_{i}$ and let

$$
d: \coprod T_{i} \rightarrow \coprod \overline{m_{i}}
$$

be $\amalg \beta_{i}$. Give $\amalg T_{i}$ the unique total order for which $c$ and the inclusions of the $T_{i}$ are order-preserving. Define

$$
T=\left(\coprod T_{i}\right) / \sim
$$

where $\sim$ is the equivalence relation generated by

$$
t \sim t^{\prime} \text { if } t \text { and } t^{\prime} \text { are adjacent, } c(t)=c\left(t^{\prime}\right), \text { and } d(t)=d\left(t^{\prime}\right) .
$$

Then $c$ and $d$ factor through $T$ to give a diagram

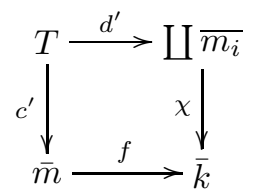


and the diagram

$$
f^{-1}(i) \stackrel{c^{\prime}}{\longleftarrow}\left(d^{\prime}\right)^{-1}\left(\overline{m_{i}}\right) \stackrel{d^{\prime}}{\longrightarrow} \overline{m_{i}}
$$

is special for each $i \in \bar{k}$. This implies that $|T|=m-k+\sum m_{i}$, so diagram (8.13) determines a diagram

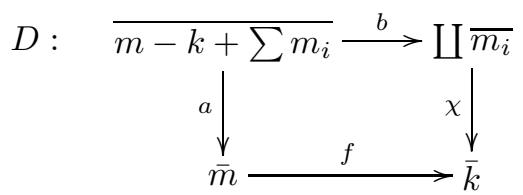

which is of type $\left(f, m_{1}, \ldots, m_{k}\right)$. Next consider the diagram

$$
\{0, \ldots, p\} \stackrel{\phi^{\prime}}{\longleftarrow} \amalg T_{i} \stackrel{\psi^{\prime}}{\longrightarrow} T
$$

where $\phi^{\prime}$ restricts on $T_{i}$ to $\alpha \circ \alpha_{i}$ and $\psi^{\prime}$ is the quotient map. If $q$ and $q^{\prime}$ are adjacent elements of $\coprod T_{i}$, then $\phi^{\prime}(q)=\phi^{\prime}\left(q^{\prime}\right) \Rightarrow \psi(q) \neq \psi\left(q^{\prime}\right)$ and $\psi^{\prime}(q)=\psi^{\prime}\left(q^{\prime}\right) \Rightarrow \phi(q) \neq$ $\phi\left(q^{\prime}\right)$; since $\left|\coprod T_{i}\right|=p+|T|$, this implies that (8.14) is special, and using the unique ordered bijections $\amalg T_{i} \rightarrow \overline{p+m-k+\sum m_{i}}$ and $|T| \rightarrow \overline{m-k+\sum m_{i}}$, we obtain a special diagram

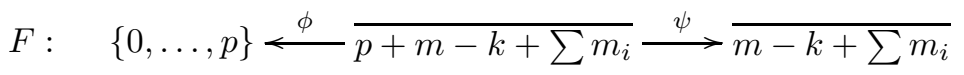

and this diagram satisfies (8.8). Thus the pair $(D, F)$ is in $\mathbf{S}_{2}$ and we may define

$$
\Phi\left(E, E_{1}, \ldots, E_{k}\right)=(D, F) .
$$

\section{REFERENCES}

[1] Benson, D.J., Representations and Cohomology II: Cohomology of Groups and Modules. Cambridge University Press 1991. MR 93g:20099

[2] Berger, C., Combinatorial models for real configuration spaces and $E_{n}$-operads. Operads: Proceedings of Renaissance Conferences (Hartford, CT/Luminy, 1995), 37-52, Contemp. Math., 202, Amer. Math. Soc., Providence, RI, 1997. MR 98j:18014

[3] Berger, C. and Fresse, B., Combinatorial operad actions on cochains. Preprint available at http://front.math.ucdavis.edu/math.AT/0109158

[4] Boardman, J.M. and Vogt, R.M., Homotopy-everything $H$-spaces. Bull. Amer. Math. Soc. 74 (1968), 1117-1122. MR 38:5215

[5] Deligne, P., Letter to Stasheff et al. May 17, 1993.

[6] Dold, A., Über die Steenrodschen Kohomologieoperationen. Annals of Math. (2) 73 (1961), 258-294. MR 23:A646

[7] Dold, A., Lectures on Algebraic Topology. Springer-Verlag, Berlin-New York, 1972. MR 54:3685

[8] Getzler, E., Cartan homotopy formulas and the Gauss-Manin connection in cyclic homology. Quantum deformations of algebras and their representations (Ramat-Gan, 1991/1992; Rehovot, 1991/1992), 65-78, Israel Math. Conf. Proc., 7, Bar-Ilan Univ., Ramat Gan, 1993. MR 95c: 19002

[9] Hinich, V.A. and Schechtman, V.V., On homotopy limit of homotopy algebras. $K$-theory, arithmetic and geometry (Moscow, 1984-1986), 240-264, Lecture Notes in Mathematics, Volume 1289, Springer-Verlag, Berlin-New York, 1987. MR 89d:55052

[10] Hirschhorn, P., Model Categories and Their Localizations. Preprint available at http://wwwmath.mit.edu/ psh/

[11] Kadeishvili, T., The structure of the $A(\infty)$-algebra, and the Hochschild and Harrison cohomologies. Trudy Tbiliss. Mat. Inst. Razmadze Akad. Nauk Gruzin. SSR 91 (1988), 19-27. MR 91a:18016 
[12] Kontsevich, M., Operads and Motives in Deformation Quantization. Lett. Math. Phys. 48 (1999), 35-72 MR 2000j:53119

[13] Kontsevich, M. and Soibelman, Y., Deformations of algebras over operads and the Deligne conjecture. Conférence Moshé Flato 1999, Volume I, 255-307, Math. Phys. Stud. 22, Kluwer Acad. Publ., Dordrecht, 2000. MR 2002e:18012

[14] Kriz, I. and May, J.P., Operads, algebras, modules and motives. Asterisque 233 (1995). MR 96j:18006

[15] Mandell, M. A., $E_{\infty}$ algebras and p-adic homotopy theory. Topology 40 (2001), 43-94. MR 2001m:55025

[16] May, J.P., The geometry of iterated loop spaces. Lectures Notes in Mathematics, Volume 271. Springer-Verlag, Berlin-New York, 1972. MR 54:8623b

[17] McClure, J.E. and Smith, J.H., A solution of Deligne's Hochschild cohomology conjecture. Proceedings of the JAMI conference on Homotopy Theory. Contemp. Math. 293 (2002), 153193.

[18] McClure, J.E. and Smith J.H., Cosimplicial objects and little $n$-cubes. I. Preprint available at http://front.math.ucdavis.edu/math.QA/0211368

[19] Smith, J.H., Simplicial group models for $\Omega^{n} S^{n} X$. Israel J. Math. 66 (1989), 330-350. MR 91e:55014

[20] Steenrod, N.E., Products of cocycles and extensions of mappings. Annals of Mathematics (2) 48 (1947), 290-320. MR 9:154a

[21] Tamarkin, D., Another proof of M. Kontsevich formality theorem. Preprint available at http://front.math.ucdavis.edu/math.QA/9803025

[22] Tamarkin, D., Formality of Chain Operad of Small Squares. Preprint available at http://front.math.ucdavis.edu/math.QA/9809164

[23] Voronov, A., Homotopy Gerstenhaber algebras. Conférence Moshé Flato 1999, Volume II, 307-331, Math. Phys. Stud. 22, Kluwer Acad. Publ., Dordrecht, 2000. MR 2002d:55009

Department of Mathematics, Purdue University, 150 N. University Street, West LAFAYETTE, INDIANA 47907-2067

E-mail address: mcclure@math.purdue.edu

Department of Mathematics, Purdue University, 150 N. University Street, West LAFAYETTE, INDIANA 47907-2067

E-mail address: jhs@math.purdue.edu 